\title{
Using Advanced Differential Interference Contrast Microscopy for High-Resolution Mapping Two-Dimensional Phase Distribution in Cells and Tissue Structures
}

\author{
Michael Shribak ${ }^{1}$ \\ 1. Marine Biological Laboratory, Woods Hole, MA, USA. \\ * Corresponding author:mshribak@gmail.com
}

The differential interference contrast (DIC) offers significant advantages for phase imaging in comparison to other currently available quantitative phase microscopy (QPM) techniques. It uses unrestricted full numerical aperture (NA) of the illumination and imaging beams and therefore provides the highest lateral and axial resolutions, and has the lowest light energy lost and the shortest exposure time. Because of wide-spectrum non-coherent illumination the image does not suffer from speckle noise. The user can choose a spectral range that is most suitable for the specimen. The optical image subtraction of two slightly different wavefronts allows observation of deep layers in scattering specimen.

We report about new developments of advanced DIC, which is called the quantitative orientationindependent differential interference contrast (OI-DIC) microscopy [1-3]. In microscope Olympus BX61 regular DIC sliders are replaced with compact two-prism OI-DIC assemblies. The OI-DIC assemblies are computer controlled, allowing rapid selection of beam shear direction and bias. The microscope does not require any modification and it can be combined with all available imaging modalities.

We compared resolutions of OI-DIC and bright-field microscopy techniques. For this purpose we used phase and amplitude Siemens Star test targets. The phase structure was created in a $90 \mathrm{~nm}$ thick $\mathrm{SiO}_{2}$ film deposited on a coverslip. The film was treated to remove the $\mathrm{SiO}_{2}$ from regions exposed to the electron beam by microlithography. The amplitude target was fabricated in a $100 \mathrm{~nm}$ thick layer of gold on a microscope slide by focused ion beam milling. Each Siemens Star consists of 36 wedge pairs. The grating period the both Siemens Stars is decreases towards the smallest period of $\sim 100 \mathrm{~nm}$ at the inner circle edge. The both, OI-DIC and bright-field images, are displayed in Fig. 1a. The bottom part of the montage represents the bright-field image. The top part shows the OI-DIC map, which was processed according to the inverse Riesz transform [3]. We used Olympus 40x/0.75NA objective lens and light with wavelength 546nm. A theoretical Abbe resolution limit was $495 \mathrm{~nm}$. We measured the intensity along medians of a bright and dark wedge pair, $m_{b}$ and $m_{d}$. Fig. $1 \mathrm{~b}$ displays dependences of the contrast in the bright-field and the OI-DIC images on the grating period. The star pattern became unresolved at period of $460 \mathrm{~nm}$ in the OI-DIC image and at period of $590 \mathrm{~nm}$ in the bright-field image. The result shows that resolution of the OI-DIC exceeds theoretical resolution limit and bright-field resolution.

The DIC microscopy technique is well known for its outstanding optical sectioning, which can be also called the depth discrimination. The OI-DIC microscope has considerably stronger optical sectioning. An example of through-focus image stack of stroma collagen fibers in cancer tissue shows that OI-DIC technique can discriminate structures $100 \mathrm{~nm}$ apart in the axial direction [4].

The OI-DIC processing algorithm employs ratios between intensities of raw DIC images. Therefore it suppresses contributions of absorption by the specimen or from nonuniformity of illumination, which can otherwise deteriorate a DIC image. The OI-DIC can produce high-quality phase images in specimen with strong absorption. Figure 2 shows conventional DIC and OI-DIC images of the hematoxylin-eosin 
(H\&E) stained pancreatic tissue, which were taken with a green filter. Because of staining the specimen has red color and the specimen structures become dark due to significant absorption in the green-blue spectral region. So the specimen structures are hardly visible in conventional image (Fig. 2a). But they are clearly present in OI-DIC image (Fig. 2b) [5].

\section{References:}

[1] M Shribak and S Inoué, Applied Optics 45 (2006), p. 460.

[2] M Shribak, The Journal of the Optical Society of America A 30 (2013), p. 769.

[3] M Shribak, KG Larkin and D Biggs, Journal of Biomedical Optics 22 (2017), p. 16006.

[4] Figshare, https://figshare.com/articles/OI-DIC_Riesz_tif/3478898 (accessed February 21, 2019).

[5] This publication was made possible by Grant Number R01-GM101701 from the NIGMS/NIH.

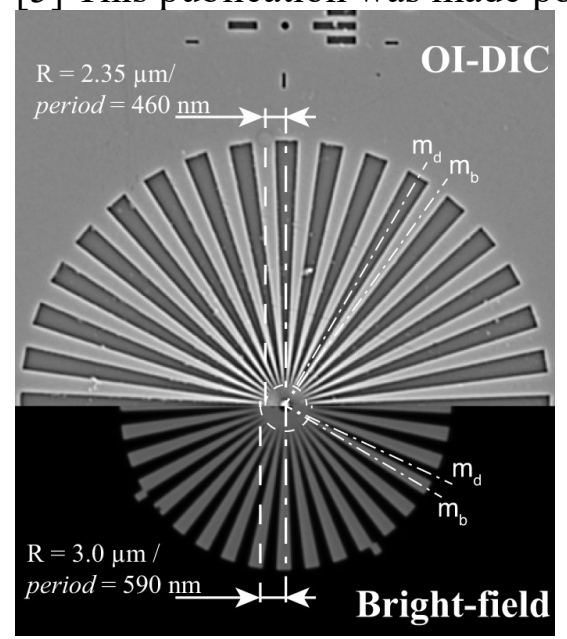

(a)

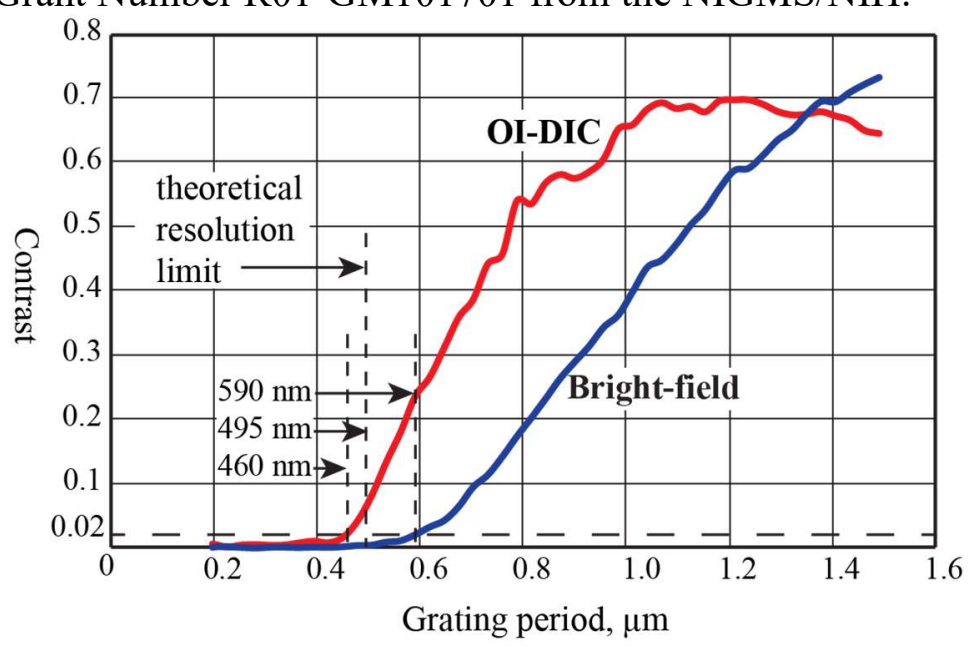

(b)

Figure 1. (a) OI-DIC and bright-field images of phase and amplitude Siemens Star resolution test targets. (b) Dependences of the contrast in the bright-field and the OI-DIC images on the grating period.

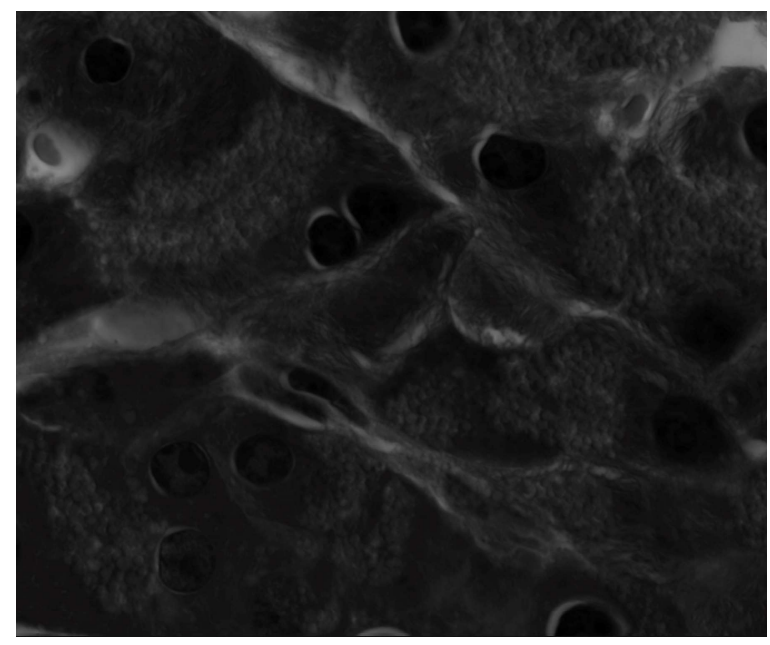

(a)

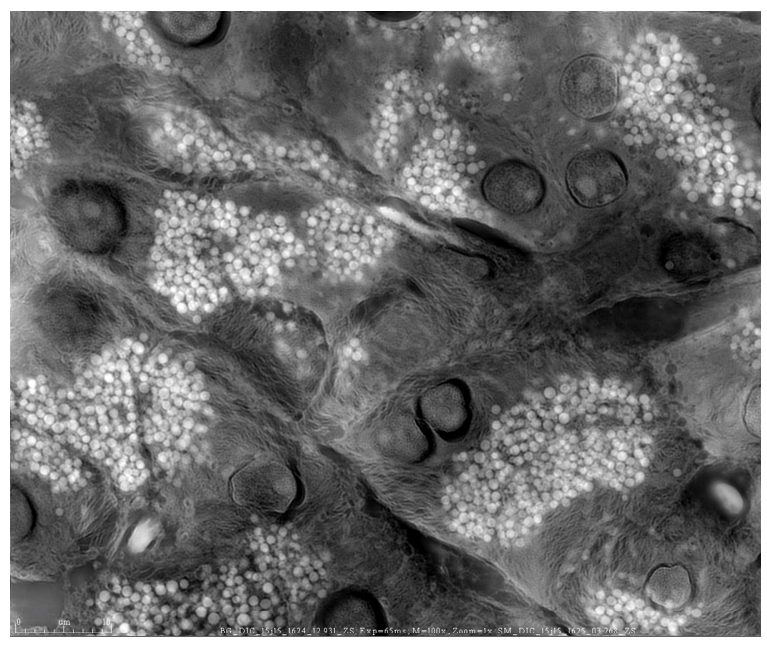

(b)

Figure 2. Image of $H \& E$ stained pancreatic tissue at wavelength $546 \mathrm{~nm}$. Image size $90 \mu \mathrm{m} \times 67 \mu \mathrm{m}$. (a) Regular DIC microscopy. Image shows strong absorption due to staining. (b) OI-DIC microscopy. 